\title{
Science with Herschel and ALMA: complementarity with ELTs
}

\author{
Tommy Wiklind \\ European Space Agency Space Telescope Operations Division/STScI, 3700 San Martin Dr., \\ Baltimore MD 21218, USA \\ email: wiklind@stsci.edu
}

\begin{abstract}
A description of the Herschel Space Observatory and the Atacama Large Millimeter Array is presented. Their scientific potential and possible synergistic effects with ELTs is discussed. Herschel is a space-based far-infrared and submillimeter telescope to be launched in 2008 and will offer 3 years of routine science operations. ALMA will become fully operational around 2012 and will co-exist with ELTs. The synergy of being able to observe the same objects with similar angular resolution and sensitivity at long and short wavelengths will contribute to our understanding of astrophysical objects and processes in general.
\end{abstract}

Keywords. telescopes, space vehicles: instrumentation, instrumentation: detectors, instrumentation: interferometers

\section{Introduction}

The next decade will see a dramatic increase in our ability to study the universe. Both through new ground-based as well as space-based facilities. While the planned instruments will not open up new unobserved wavelength windows, they will bring both sensitivity and angular resolution to parts of the electromagnetic spectrum where our knowledge is so far based on rather coarse observations.

Among the planned instruments aimed at the infrared, far-infrared and submillimeter, are the James Webb Space Telescope (JWST), the Herschel Space Observatory and the Atacama Large Millimeter Array (ALMA). In addition, Planck and Astro-F will observe at far-infrared wavelengths, albeit with a lower angular resolution.

In addition to the planned instruments, there are existing facilities, such as groundbased optical/near-infrared 8-10m telescopes, and the Hubble Space Telescope (HST), observing from the UV to the near-infrared. The combination of existing facilities and the planned telescopes provide a complete wavelength coverage from long wavelength radio to the UV. This is illustrated in Fig. 1, where we can also see that while the angular resolutions achieved at $\mathrm{UV} /$ near-infrared will be comparable to that achieved with ALMA at $\mathrm{mm} /$ submillimeter wavelengths, the same is not true at intermediate far-infrared wavelengths.

Not all of the planned, or existing, telescopes and instruments will be co-existing. Especially the space-based telescopes have a limited life time (Herschel), or a launch date at 2014 or later (JWST). In some cases this is beneficial, as in the case with the launch in 2006 of Astro-F which will do an all-sky survey in the far-infrared with significantly higher sensitivity than IRAS, but with comparable angular resolution. In Fig. 2 the time lines of a few facilities are shown. With the present time lines for the ELT projects, there will be no far-infrared, nor space-based submillimeter facilities available when these become operational. In order to facilitate complementarity between the ELT and Herschel, careful planning has to be done at the present. 


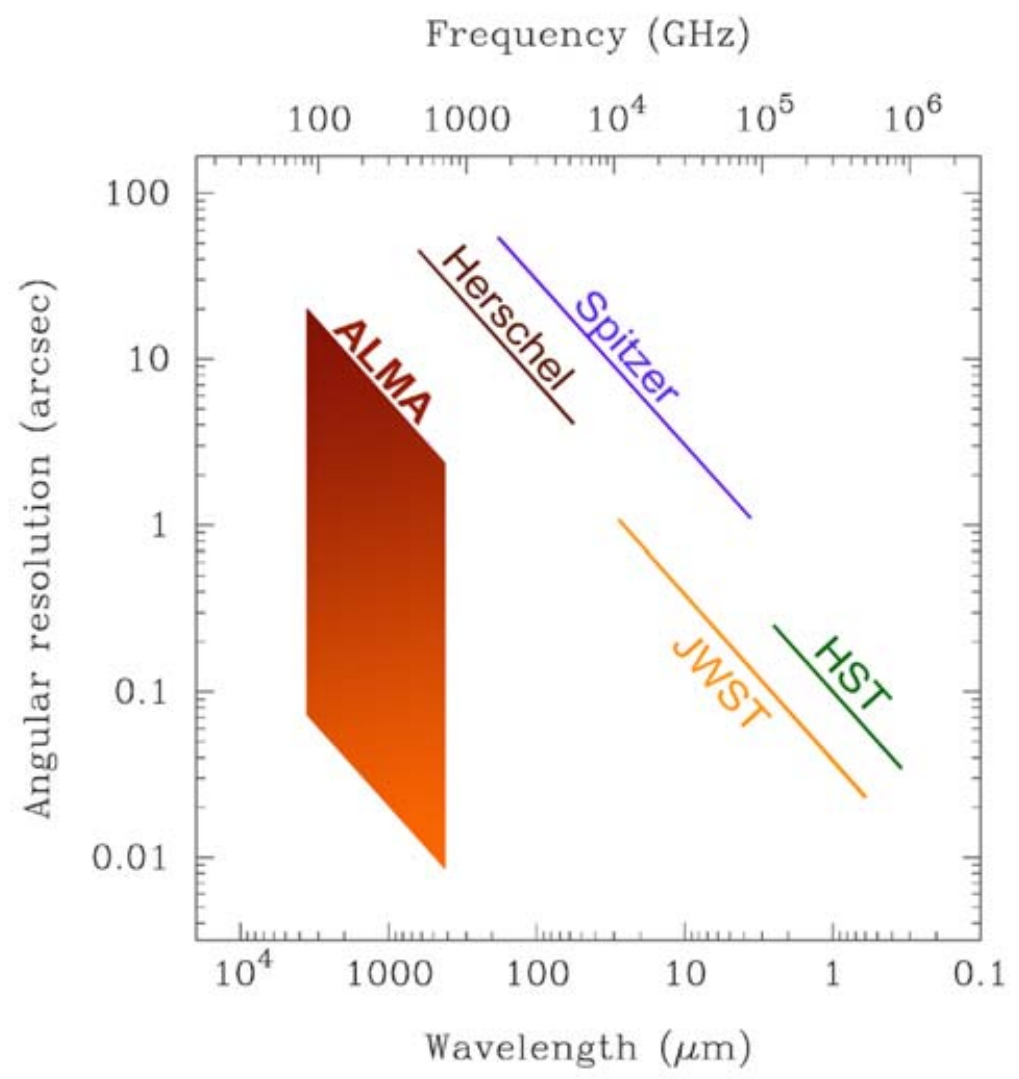

Figure 1. Angular resolution as a function of wavelength for ALMA and a few space-based telescopes. While sub-arcsecond resolution will be achieved at $\mathrm{mm} /$ submillimeter and optical/ near-infrared wavelengths, the far-infrared part will only be accessible with resolutions of a few arcseconds. The planned ELTs will fall in the lower right corner of the figure, with angular resolutions comparable to present day space-based missions.

\section{The Herschel Space Observatory}

The Herschel Space Observatory is a far-infrared/submillimeter telescope that will observe in the $60-670 \mu \mathrm{m}$ wavelength range. Herschel is the fourth of the original ESA's Horizon 2000 cornerstone missions. It will be launched together with the Planck satellite on an Ariane 5 ECA from Kouro. Launch is planned for 2008. Herschel and Planck will separate from each other immediately after launch and then independently injected into transfer orbit to the second Langrangian point (L2). See Pilbratt (2005) for a detailed description of Herschel and its subsystems.

The telescope on-board Herschel is a $3.5 \mathrm{~m}$ primary classical Cassegrain with an undersized secondary. The primary is constructed out of silicon carbide ( $\mathrm{SiC}$ ) and is diffration limited at $\lambda<90 \mu \mathrm{m}$. The telescope assembly is passively cooled to about $80 \mathrm{~K}$, with a uniform and slowly changing temperature distribution. All mirror alignments will be done prior to launch as there are no possibilities for in-flight adjustments of the optics, including focusing. The spacecraft has a cryostat with superfluid helium to provide cooling to the detectors. Herschel will provide a minimum of 3 years of observations once routine operation is started. About $2 / 3$ of the time will be open to the general astronomical community through standard proposal procedures. 


\section{Time plan for major space based observatories and ALMA (Nov 2005)}

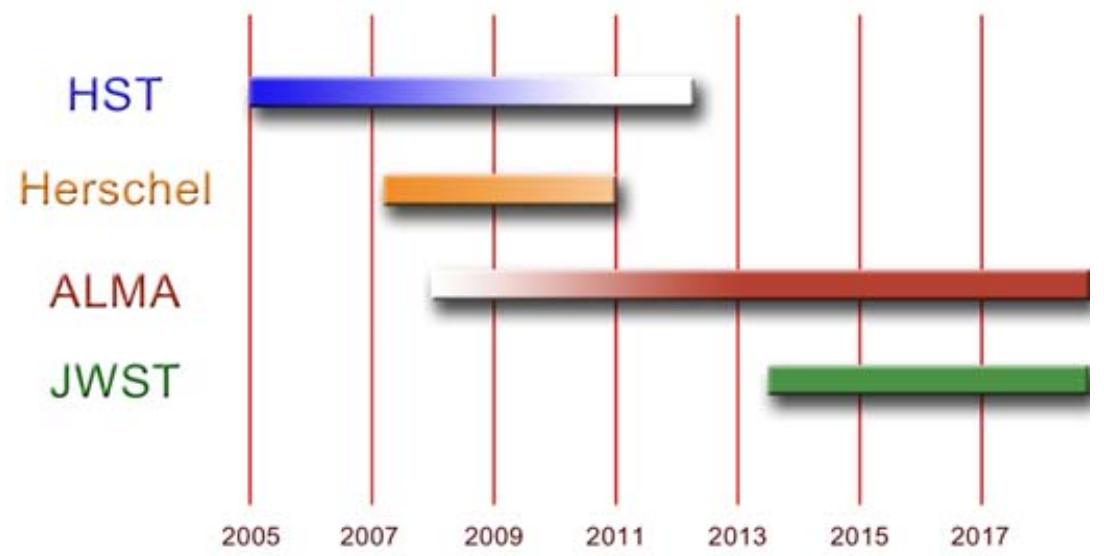

Figure 2. The expected time lines for ALMA, Herschel, JWST and HST. The actual dates for starting/ending of these missions are uncertain and is indicted by a 'diffuse' coloring. It seems certain, though, that the Herschel Space Observatory will only partly overlap in time with either ALMA, JWST, or the HST.

\subsection{Instrumentation}

Herschel will carry three instrument packages, built by consortia and briefly presented below. For more details and further references see Pilbratt (2005).

\subsubsection{The Photodetector Array Camera and Spectrometer (PACS)}

The PI for this instrument is A. Poglitsch, MPE, Garching, Germany. PACS will cover the shortest wavelength band offered by Herschel, $60-210 \mu \mathrm{m}$. As the name implies, it is both an imaging and spectrometric instrument. The detector is a bolometer array of $64 \times 32$ pixels, covering a field-of-view of $1^{\prime} .75 \times 3^{\prime} .5$.

There are two basic modes of operation. Imaging dual-band photometry with either of the two short wavelength bands at $60-85 \mu \mathrm{m}$ or $85-130 \mu \mathrm{m}$ bands working simultaneously with the long wavelength band $130-210 \mu \mathrm{m}$. Both the short and long wavelength bands see the same part of the sky. In addition to the photometer, PACS contains an integralfield spectrograph operating in the $57-210 \mu \mathrm{m}$ range. The spectrograph consists of a $5 \times 5$ pixel detector with a field-of-view of $47^{\prime \prime} \times 47^{\prime \prime}$. The bandwidth is $\sim 1500 \mathrm{~km} \mathrm{~s}^{-1}$ with a spectral resolution $\sim 175 \mathrm{~km} \mathrm{~s}^{-1}$, i.e. $\mathrm{R} \sim 1700$ at $150 \mu \mathrm{m}$. The spectrometer fields are offset from the photometric fields and can not be used together with the photometric mode. For further details, see Poglitsch et al. (2004).

\subsubsection{The Spectral and Photometric Imaging Receiver (SPIRE)}

The PI for this instrument is M. Griffin at the University of Wales, Cardiff, UK. SPIRE will cover the wavelength range $200-670 \mu \mathrm{m}$ and consists of a three-band imaging photometer (bolometer arrays) and an imaging Fourier Transform Spectrometer, also using bolometer detectors. The imaging is done in three bands, 250, 360 and $520 \mu \mathrm{m}$, with a field-of-view of $4^{\prime} \times 8^{\prime}$. The three bands use different bolometer arrays but see the same part of the sky and can be used simultaneously. In order to produce fully sampled images, the observations need to be done in a jiggle-mode. The angular resolution is, 
$18^{\prime \prime}, 25^{\prime \prime}$ and $36^{\prime \prime}$ for the three bands, respectively. The spectrometer operates in the wavelength range $200-670 \mu \mathrm{m}$. The resolving power ranges from $\mathrm{R} \sim 20-1000$ at a wavelength of $250 \mu \mathrm{m}$. The field-of-view of the spectrometer is circular with a diameter of $2^{\prime} .6$. See Griffin et al. (2004) for further details on SPIRE.

\subsubsection{The Heterodyne Instrument for the Far Infrared (HIFI)}

The PI for this instrument is Th. de Graauw, SRON, Groningen, the Netherlands. The operating wavelength ranges between $157-625 \mu \mathrm{m}$. While PACS and SPIRES cover the $57-670 \mu \mathrm{m}$ range with imaging and low-to-medium resolution spectrographs, HIFI offers extremely high spectral resolution over a slightly smaller wavelength range. The high resolution is due to the fact that HIFI is a heterodyne system with two backends; an Acousto-Optical Spectrometer (AOS) and an autocorrelator. The wavelength coverage is divided into six bands and HIFI has a spectral resolution of $140-280 \mathrm{kHz}$, corresponding to $\sim 0.03-0.07 \mathrm{~km} \mathrm{~s}^{-1}$ at $240 \mu \mathrm{m}$. The instantaneous bandwidth is $4 \mathrm{GHz}$ with two polarizations. The detectors are near the quantum noise limit. The angular resolution will range from $13^{\prime \prime}$ to $39^{\prime \prime}$.

\subsection{Herschel Science}

Herschel is equipped to observe a wide variety of objects. The wavelength coverage is optimal for studying the cool components of galaxies, stars as well as planets. Black body emitters with temperatures in the range $10-50 \mathrm{~K}$ will peak within the wavelengths observable with Herschel. The atomic and interstellar medium is characterized by temperatures from just a few $K$ up to a few hundred $K$, and will be accessible through continuum and a large number of molecular as well as atomic fine-structure lines. Only a few high-lights of the potential science can be presented here. A recent compilation of science issues for Herschel (as well as for ALMA), can be found in The Dusty and Molecular Universe (ed. Wilson 2005).

With its coverage of the far-infrared submillimeter Herschel will be a unique instrument. The wavelengths available through Herschel will overlap with ALMA and Spitzer (see Fig. 1). However, Herschel will provide both better angular resolution and better sensitivity than Spitzer and will cover wavelengths too short for ALMA.

The science potential of Herschel can be divided into three, non-exhaustive, main areas where the scientific impact is likely to be very high:

- Deep galactic/extragalactic broad band photometric surveys in the range 100$600 \mu \mathrm{m}$

(a) far-infrared bright galaxies at high redshift

(b) AGN vs. star formation heating of Ultraluminous infrared bright galaxies (ULIRGs)

(c) Molecular clouds and dust in the Milky Way

- Spectroscopy of infrared fine-structure lines

(a) important cooling lines for the interstellar medium

(b) AGN vs. star formation as a source of far-infrared emission

- High resolution spectroscopy of interstellar and circumstellar molecules

(a) astrochemistry

(b) physical parameters of star forming regions ( $\mathrm{T}, \rho, \mathrm{v}_{\mathrm{r}}, \Delta v$ )

(c) dust and metal production mechanisms and rates

(d) protostellar disks and embedded protostars

(e) planetary atmospheres in the solar system

(f) comets 
The main instruments for the deep extragalactic surveys are the photometers of PACS and SPIRES. All three spectrographs are likely to contribute to the fine-structure line studies, while HIFI will explore molecular lines which have not previously been available. For instance, many light hydride molecules are important for our understanding of astrochemistry, but since they are light, they have their lowest rotational transitions at high frequency. HIFI will make it possible to observe these ISM constituents for the first time.

One potential problem for surveys of far-infrared objects is the limited angular resolution of Herschel, leading to confusion of weak far-infrared emitting objects. In Fig. 4 the observed flux density of far-infrared bright objects is plotted as a function of redshift. In the left panel the observed flux density with PACS, at $130-210 \mu \mathrm{m}$ is shown for objects with a total far-infrared luminosity of $10^{12}$ and $10^{11} \mathrm{~L}_{\odot}$. The horizontal line represents the $5 \sigma$ detection limit for a point-like object after 1 hour of on-source integration. The lines correspond to different dust temperatures. While PACS will be able to detect objects characterized by warm dust out to redshifts of $z \sim 3$ for $\mathrm{L}_{\mathrm{FIR}} \sim 10^{12} \mathrm{~L}_{\odot}$, this is limited to $z \leqslant 1.5$ for objects with $\mathrm{L}_{\mathrm{FIR}} \leqslant 10^{11} \mathrm{~L}_{\odot}$. Hence, confusion may not be a problem for extragalactic surveys, unless they go much deeper than $\sim 2$ mJy.

\section{The Atacama Large Millimeter Array}

The Atacama Large Millimeter Array (ALMA) is a truly international astronomical collaboration, involving more than 15 countries, affiliated with either the European side, through the European Southern Observatory (ESO), the North American side, through Associated Universities Inc (AUI) via the National Radio Astronomical Observatory (NRAO), or the Japanese ALMA-J side via National Institutes of Natural Sciences (NINS).

The aim of ALMA is to bring the sensitivity and angular resolution of millimeter and submillimeter observations in par with that achieved on the optical and near-infrared side, or better. In order to do this, the original design of ALMA called for $6412 \mathrm{~m}$ antennas, located in a high and dry site, and allowing baselines of $10-15 \mathrm{~km}$. The Chajnantor plateau, east of San Pedro de Atacama in Chile, was chosen as the ALMA site. It has exceptional atmospheric transparency in the submillimeter and is flat enough to permit long baselines as well as easy access and movement of the antennas between stations. The maximum angular resolution will be $\sim 10$ milliarcsec at the highest frequency and the longest baselines (see Fig. 1). The design of ALMA is defined by a number of 'Level 1' science requirement, i.e. specification that the instrument must be able to fulfill. These are:

(a) The ability to detect spectral line emission from $\mathrm{CO}$ or [CII] in a normal galaxy like the Milky Way at $z=3$ in less than 24 hours of observation.

(b) The ability to image the gas kinematics in protostars and protoplanetary disks around young stars at a distance of $150 \mathrm{pc}$ (roughly the distance to star-forming clouds in Oph and $\mathrm{CrA}$ ), enabling one to study their physical, chemical, and magnetic field structures and to detect the gaps created by planets undergoing formation in the disks.

(c) The ability to provide precise images at an angular resolution of 0 ". 1 . Here the term precise image means accurately representing the sky brightness at all points where the brightness is greater than $0.1 \%$ of the peak image brightness. This requirement applies to all sources visible to ALMA that transits at an elevation greater than 20 degrees.

While the first requirement is essentially dependent on the total collecting area, i.e. the number of telescopes in the array, the second and third requirements put stringent limits 

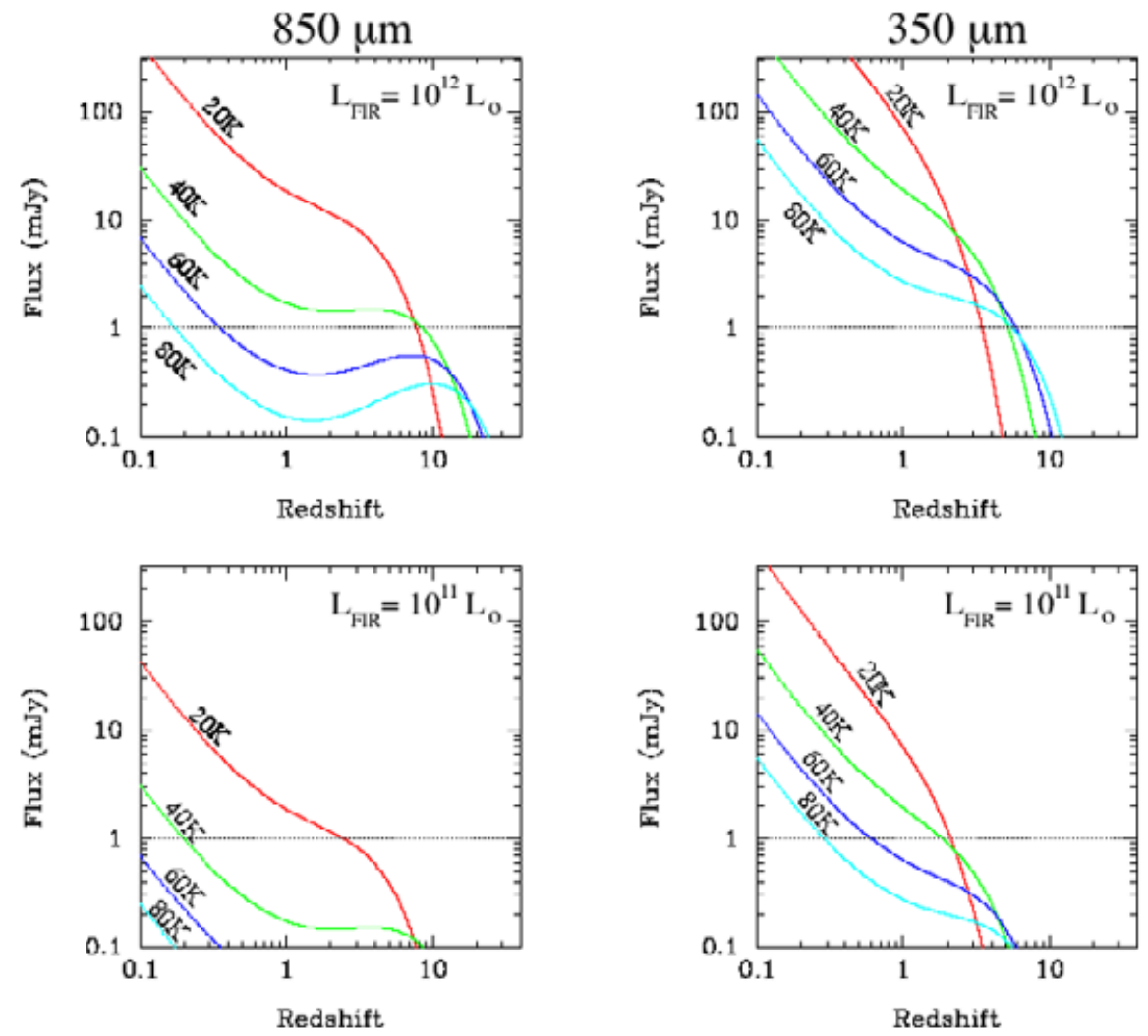

Figure 3. The observed flux densities as a function of redshift for far-infrared luminous objects with a spectral energy distribution (SED) characterized by different dust temperatures. The SED is modeled as a modified blackbody function $S_{\nu} \propto B_{\nu}\left(T_{d}\right)(1-\exp (\tau))$, where $\tau=\left(\nu / \nu_{0}\right)^{\beta}$ and $\beta=1.5, \nu_{0}=5 \times 10^{12} \mathrm{~Hz}(60 \mu \mathrm{m})$. Observations at two wavelengths are depicted $(850 \mu \mathrm{m}$ and $350 \mu \mathrm{m})$, and two total far-infrared luminosities $\left(10^{12}\right.$ and $\left.10^{11} \mathrm{~L}_{\odot}\right)$. The horizontal line at $1 \mathrm{mJy}$ is the approximate limit for existing telescopes. This sensitivity allows observation of cool $\left(\mathrm{T}_{d} \leqslant 40-50 \mathrm{~K}\right)$ and far-infrared luminous $\left(\sim 10^{12} \mathrm{~L}_{\odot}\right)$ objects out to redshifts $z=5-10$, depending on the observed wavelength.

on the spectral and angular resolution as well as the necessary calibration accuracy. The latter depends on the stability of the atmosphere, or how well it is possible to measure and correct for changes in its transparency at the observed frequency. Precision imaging requires very good calibration of both phase and amplitude of the signal, both being notoriously difficult to measure to better than $10-20 \%$ at millimeter/submillimeter wavelengths. ALMA, on the other hand, will try to do this with an accuracy better than a few percent. This will be achieved through a combination of fast switching between source and calibrators, correcting for fluctuations on the 10-30 second level, and through the use of water vapor radiometers to measure phase fluctuations on the time scale of seconds.

Recent developments within the ALMA project have called for a revision of the number of antennas that, at least initially, will be constructed. Instead of the planned 64 antennas, ALMA will be made up of $5012 \mathrm{~m}$ antennas, with the option to extend to the full 64 antennas at a later date. The Japanese contribution to ALMA will add $312 \mathrm{~m}$ and $127 \mathrm{~m}$ antennas, making up the ALMA Compact Array (ACA). The compact array is needed in 

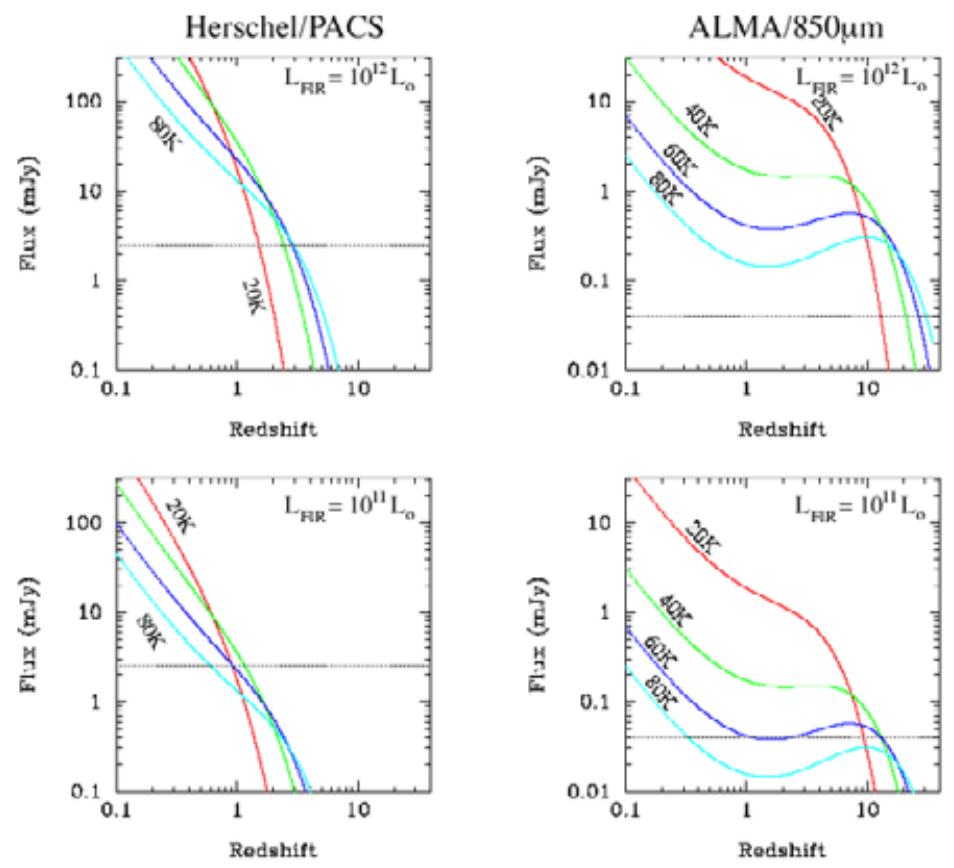

Figure 4. Similar plot as in Fig. 3, but now showing the actual sensitivity of the PACS $(130-210 \mu \mathrm{m})$ instrument on-board Herschel (left panels), and band $3(850 \mu \mathrm{m})$ on ALMA (right panels). The horizontal lines correspond to the $5 \sigma$ sensitivity limit for a point source and 1 hour of observation. With these restrictions, PACS will detect sources out to redshifts $z \approx 3-4$ for far-infrared luminous objects, while ALMA will be able to observe the same type of sources to redshifts $z>10$. There is, however, a strong temperature dependence.

order to reach high dynamic range over a large field-of-view, i.e. a well sampled $u v$-plane. The reduction in scope of the ALMA instrument means that the sensitivity will be $\sim 78 \%$ of the initial plans. Sensitivity is approximately directly proportional to the number of antennas, while integration times goes as $\sim N^{-2}$. With the re-scaled ALMA, integration times need to be increased by $\sim 1.65$ in order to reach the same sensitivity as with the earlier version. After careful consideration of the impact on the science goals, especially the Level-1 requirements, it appears that most Primary Science Goals will be reach with the smaller ALMA.

\subsection{ALMA Science}

ALMA was designed from the start to be a versatile instrument, capable of providing sensitive and detailed information on most objects of astrophysical interest. The width of the scientific potential can be seen through the Design Reference Science Plan (DRSP), where a number of astronomers where asked to write 'real' proposals for ALMA (although without having the possibility of being granted observing time) $\dagger$.

As with Herschel, it is not possible to discuss all of the potential science that can be done with ALMA. There have been several international meetings discussing various aspects of the science potential of ALMA (eds: Shaver 1996; Wotten 2001; Surdej et al. 2001; Wilson 2005). Below is a non-exhaustive list of main areas where ALMA is likely to make a fundamental impact. The list is based on the DRSP.

$\dagger$ The DRSP can be accessed at http://www.strw.leidenuniv.nl/ alma/drsp.html 


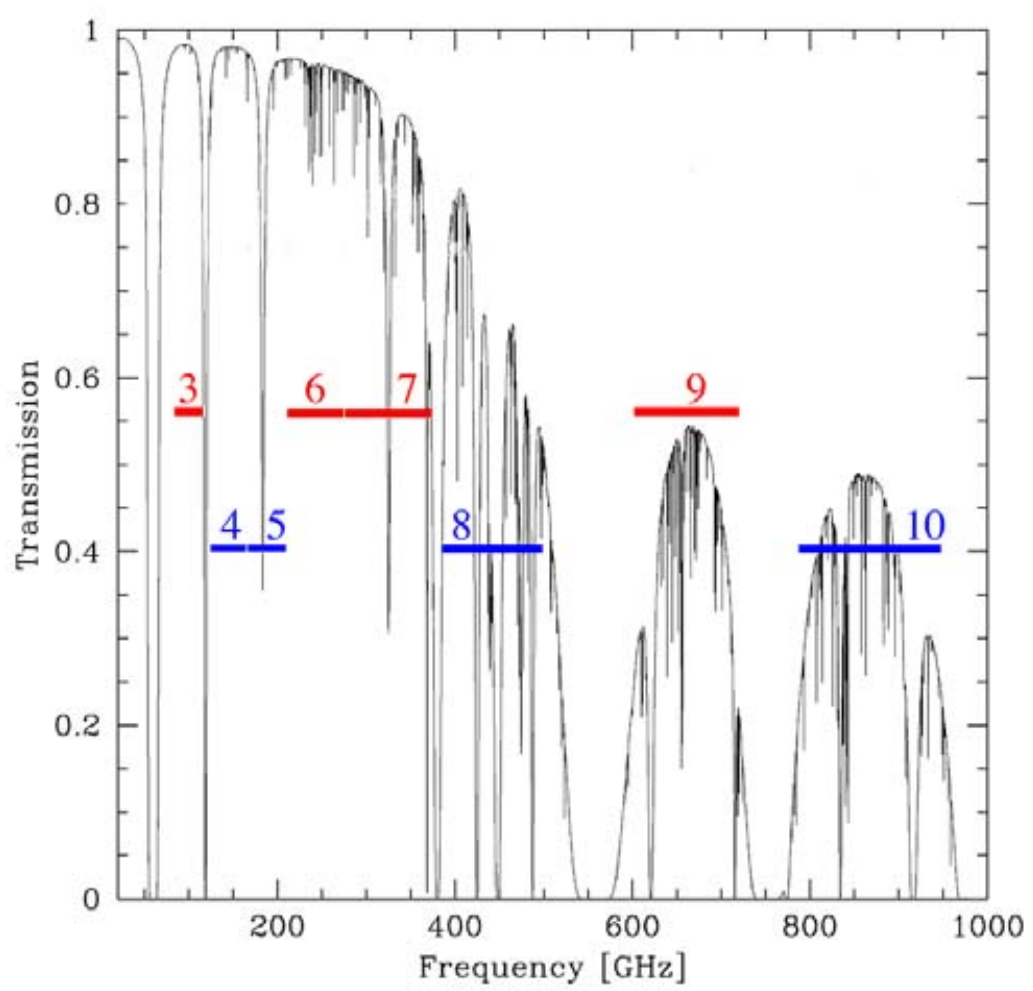

Figure 5. Atmospheric transmission at the Chajnantor site with a precipital water vapor content of $0.5 \mathrm{~mm}$. The ALMA frequency bands are indicated. The first set of receivers to be implemented will cover band 3,6,7 and 9, with a possibility for additional bands either later on, or through ALMA-J. Band 7 is the optimal frequency band for detection of dust continuum emission. Band 1 and 2 (not depicted) represent the $30 \mathrm{GHz}$ band, specifically for the Sunyaev-Zeldovich effect, and the low-frequency part of the $3 \mathrm{~mm}$ window $(70-86 \mathrm{GHz})$, respectively.

- Galaxies and cosmology

(a) Detect dust continuum to $z \sim 10$

(b) Redshift distribution through blind line surveys

(c) Dynamical mass through mapping of line emission

(d) Detailed study of Giant Molecular Clouds in low $-z$ galaxies, dynamics and chemistry

- Interstellar medium

(a) Spatially resolved molecular line surveys

(b) Protostellar collapse phase

(c) Important cooling lines (molecular and atomic fine-structure lines)

(d) Observations of many light hydride molecules

- Star and planet formation

(a) Detect debris disks

(b) Detect gaps caused by Jupiter size planets

(c) Direct detection of giant planets

- Stellar astronomy

(a) Photospheric emission from $>600$ main sequence stars 
(b) Chromospheric activity

(c) Stellar astrometry

(d) Dust formation, mass loss and chemistry of circumstellar shells

- Solar system objects

(a) Temperature, pressure, water content of planetary atmospheres (Mars, Venus)

(b) Thermal gradients and pressure in the troposphere of giant planets

(c) Chemistry and dynamics of Titan's atmosphere

(d) Imaging of volcanoes on Io

(e) Solar activity

Current single dish telescopes, equipped with bolometer arrays, and existing millimeter wavelength interferometers, can reach continuum flux densities of $\sim 1 \mathrm{mJy}$ in reasonable observing times. In Fig. 3 this sensitivity limit is compared with the observed flux density of far-infrared luminous objects as a function of redshift, dust temperature and total far-infrared luminosity. The comparison is made for two different observed frequencies, $850 \mu \mathrm{m}$ and $350 \mu \mathrm{m}$. Submillimeter observations of the dust continuum have the potential of detecting galaxies out to $z \sim 10$, where the peak of the dust emission is shifted to longer wavelengths and the observed window begins to sample the Wien part of the spectral energy distribution. This, however, is only possible for the most luminous (i.e. $\mathrm{L}_{\mathrm{FIR}} \geqslant 10^{12} \mathrm{~L}_{\odot}$ ) and coolest (i.e. $\mathrm{T}_{\mathrm{d}} \sim 40 \mathrm{~K}$ ) objects. If the dust is characterized by a slightly higher temperature, the current detection limit is insufficient to detect these objects. The situation is slightly better at an observed wavelength of $350 \mu \mathrm{m}$. However, there are no existing bolometer working at this wavelength with a sensitivity limit as low as $1 \mathrm{mJy}$. In Fig. 4 the same plot is shown for an observed wavelength of $850 \mu \mathrm{m}$, indicating the $5 \sigma$ detection limit in 1 hour of observing with ALMA. In this case, all objects with $\mathrm{T}_{\mathrm{d}} \geqslant 60 \mathrm{~K}$ and $\mathrm{L}_{\mathrm{FIR}} \sim 10^{11} \mathrm{~L}_{\odot}$ can be observed to $z=10$ or greater.

\section{Synergies with ELTs}

ALMA is likely to be the main millimeter/submillimeter facility for several decades to come and, in contrast to Herschel, ALMA will co-exist with the planned ELTs. Although the angular resolution of ALMA will be as high as 10mas, the combination of ELTs and ALMA will allow studies of the same objects in entirely different wavelength regimes, and therefore entirely different emission mechanisms, to an angular resolution set by the ELTs (i.e. a few tenths of an arcsecond). In some areas, such as protoplanetery disks, ELTs and ALMA have the same direct observational goals, i.e. imaging of gaps in the debris disk, imaging of the extended disk, outflows, etc., but using different wavelengths. In other areas, observations with ALMA and ELTs allows the physical properties of objects to be assessed through extending our understanding of how the spectral energy is distributed over wavelengths. As outlined in Fig. 1, there is a lack of high angular resolution instruments in the wavelength regime between that provided by ELTs at optical/near-infrared and ALMA at millimeter/submillimeter wavelengths. However, observations of high redshift objects will actually allow ALMA to probe the far-infrared 'hole' with angular resolution similar to that of ELTs.

It is interesting to speculate about what instruments like Herschel, ALMA and the planned ELTs can accomplish if new classes of objects are discovered. One such area may have recently been uncovered with the detection of a massive, yet old and evolved galaxy at a redshift $z=6.5$ (Mobasher et al. 2005). The formation redshift of this galaxy is at $z_{\mathrm{f}} \sim 10-15$. Broadband photometry with HST/ACS, VLT/ISAAC and Spitzer/IRAC suggest that the galaxy has a stellar mass of $5 \times 10^{11} \mathrm{M}_{\odot}$, an age of about $600 \mathrm{Myr}$, 
contains no extinction and is presently not forming stars. A recent search for similarly old and massive galaxies at $z \sim 6-7$ have shown several promising candidates. Since these galaxies are very distant and currently not forming stars, they are extraordinarily difficult to observe spectroscopically with present day instrumentation. With the JWST it might be possible to obtain spectral signatures of the aging stellar population, but the need for a large ELT is obvious. Likewise, the only instrument which may be able to observe the actual star forming event at $z_{\mathrm{f}} \sim 10-15$ is ALMA at its longest operational wavelengths.

\section{Acknowledgements}

I would like to thank Göran Pilbratt and Tom Wilson for careful reading of an earlier version of the manuscript and for providing useful comments.

\section{References}

Griffin, M.J., Swinyard, B.M. \& Vigroux, L. 2004, SPIE 5487, 413

Mobasher, B., Dickinson, M., Ferguson, H.C., Giavalisco, M., Wiklind, T. et al. 2005, ApJ 635, 832

Pilbratt, G.L. 2005, in: A. Wilson (ed.) The Dusty and Molecular Universe: A Prelude to Herschel and ALMA, ESA Publications Division, ESTEC Noordwijk, The Netherlands

Poglitsch, A., Waelkens, C., Bauer, O.H., Cepa, J., Henning, T.F., van Hoof, C., et al. 2004, SPIE 5487, 425

Shaver, P. 1996, Science with Large Millimeter Arrays, Proceedings of the ESO-IRAM-NFRAOnsala Workshop, Garching, Germany, Berlin, Springer-Verlag

Surdej, J., Swings, J.P., Caro, D. \& Detal, A. 2001, From Optical to Millimetric Interferometry: Scientific and Technological Challenges, Liège, Belgium, Université de Liège

Wilson, A. 2005, The Dusty and Molecular Universe: A Prelude to Herschel and ALMA, ESA SP-577, ESA Publications Division, ESTEC Noordwijk The Netherlands

Wotten, A. 2001, Science with the Atacama Large Millimeter Array, ASP Conf. Series, Vol 235, San Francisco, ASP

\section{Discussion}

Mould: When will ALMA see first light, and to whom do we apply for time?

WikLind: The plan is to have first light some time during 2008/2009. Early science operation will start once there are 6-8 antennas on the Chajnantor site. This will continue to grow in steps of 6-8 antennas until the whole array is operational, around 2012 . The proposal process is being discussed right now. It is likely that each partner will have their own proposal review process with a built-in mechanism to handle overlap and duplications.

DENNEFELD: It was mentioned yesterday that one possible instrument for an ELT was a SCUBA-type survey detector. Have you evaluated what would be the gain of such a survey instrument over ALMA, as a function of ELT diameter?

WIKLIND: ALMA is not a very efficient survey instrument for surveys of large areas. The field of view is limited to 10-40 arcsec, depending on the observing frequency. So, mapping will require multiple pointings. A bolometer array on an ELT would have excellent sensitivity and an angular resolution of $\sim 3-5^{\prime \prime}$. Operating in a fast mapping made, such an instrument could be a survey instrument at submillimeter wavelengths. 